\title{
High rate of HIV-I drug resistance in treatment failure patients in Taiwan, 2009-20I4
}

\author{
Hung-Chin Tsai ${ }^{1-3}$ \\ I-Tzu Chen' \\ Kuan-Sheng $\mathrm{Wu}^{1,2}$ \\ Yu-Ting Tseng' \\ Cheng-Len Sy' \\ Jui-Kuang Chen'
}

Susan Shin-Jung Lee ${ }^{1,2}$

Yao-Shen Chen ${ }^{1,2}$

'Department of Medicine, Division of Infectious Diseases, Kaohsiung Veterans General Hospital, Kaohsiung,

${ }^{2}$ Faculty of Medicine, School of

Medicine, National Yang-Ming

University, Taipei, ${ }^{3}$ Department of

Parasitology, Kaohsiung Medical

University, Kaohsiung, Taiwan
This article was published in the following Dove Press journal:

Infection and Drug Resistance

16 October 2017

Number of times this article has been viewed

Background: Drug resistance to nucleoside reverse transcriptase inhibitors (NRTIs), nonnucleoside reverse transcriptase inhibitors (NNRTIs), and protease inhibitors (PIs) has been associated with loss of viral suppression measured by a rise in HIV-1 RNA levels, a decline in CD4 cell counts, persistence on a failing treatment regimen, and lack of adherence to combination antiretroviral therapy.

Objectives: This study aimed to monitor the prevalence and risk factors associated with drug resistance in Taiwan after failure of first-line therapy.

Materials and methods: Data from the Veterans General Hospital Surveillance and Monitor Network for the period 2009-2014 were analyzed. Plasma samples from patients diagnosed with virologic failure and an HIV-1 RNA viral load $>1000$ copies/mL were analyzed by the ViroSeq ${ }^{\text {TM }}$ HIV-1 genotyping system for drug susceptibility. Hazard ratios (HRs) for drug resistance were calculated using a Cox proportional hazard model.

Results: From 2009 to 2014, 359 patients were tested for resistance. The median CD4 count and viral load (log) were 214 cells/ $\mu \mathrm{L}$ (interquartile range [IQR]: 71-367) and 4.5 (IQR: 3.9-5.0), respectively. Subtype B HIV-1 strains were found in $90 \%$ of individuals. The resistance rate to any of the three classes of antiretroviral drugs (NRTI, NNRTI, and PI) was $75.5 \%$. The percentage of NRTI, NNRTI, and PI resistance was $58.6 \%, 61.4 \%$, and $11.4 \%$, respectively. The risk factors for any class of drug resistance included age $\leq 35$ years (adjusted HR: 2.30 , CI: $1.48-3.56 ; p<0.0001$ ), initial NNRTI-based antiretroviral regimens (adjusted HR: 1.70, CI: 1.10-2.63; $p=0.018$ ), and current NNRTI-based antiretroviral regimens when treatment failure occurs (odds ratio: 4.04, CI: $2.47-6.59 ; p<0.001$ ). There was no association between HIV-1 subtype, viral load, and resistance. Conclusion: This study demonstrated a high level of resistance to NRTI and NNRTI in patients with virologic failure to first-line antiretroviral therapy despite routine viral load monitoring. Educating younger men who have sex with men to maintain good adherence is crucial, as PI use is associated with lower possibility of drug resistance.

Keywords: antiretroviral therapy, HIV, drug resistance, treatment failure

\section{Introduction}

The introduction of highly active antiretroviral therapy (HAART) has dramatically changed the disease course of HIV infection, making it a chronic and controllable disease. ${ }^{1-4}$ Suppression of virus replication can reduce disease progression and transmission. Monitoring HIV-1 drug resistance is essential for accessing requirements for new drugs and for stopping the spread of resistances. As such, patients with virologic failure provide a surrogate marker for treatment effectiveness. ${ }^{5}$ Continuing with failed regimens may lead to more complex mutation patterns, the development
Correspondence: Hung-Chin Tsai Department of Medicine, Division of Infectious Diseases, Kaohsiung Veterans General Hospital, \#386 Ta-Chung Ist Road, Kaohsiung 813, Taiwan Tel +88673422121 ext 2029 Fax +88673468292

Email hctsail0II@yahoo.com.tw 
of cross-resistance, and the forward transmission of drugresistant HIV to antiretroviral therapy (ART)-naïve patients. ${ }^{6}$

The World Health Organization recommends monitoring for acquired HIV drug resistance in individuals receiving ART. ${ }^{7}$ A recent review reported high drug resistance levels in patients with virologic failure and the most common resistance mutations, M184V and K103N, were found in $65 \%$ and $52 \%$ of patients, respectively. ${ }^{8}$ However, the prevalence rate of acquired ART resistance in Asia, where routine viral load monitoring is available, remains unknown.

Genotypic drug resistance testing (GRT) before treatment can influence the rate of acquired drug resistance mutation. In the Swiss HIV Cohort Study, $28.9 \%$ of post-ART patients had detectable drug resistance mutation, whereas in the recent ART-initiator group, only 45 of 2092 (1.6\%) patients with pretreatment GRT acquired drug resistance mutations on ART. ${ }^{9}$ Thus, regular surveillance of transmitted drug resistance is important to combat drug resistance.

The prevalence of transmitted HIV resistance had recently increased to $5 \%$ in some areas of South Africa, Kenya, and Zambia, and up to $15 \%$ in Uganda. ${ }^{10-12}$ In Asia, this rate was around $4 \%-12 \%$, including $3.8 \%$ in China, ${ }^{13} 7.7 \%$ in Japan, ${ }^{14} 12 \%$ in South Korea, ${ }^{15} 4.9 \%$ in Thailand, ${ }^{16}$ and $8 \%-11.1 \%$ in northern Taiwan. ${ }^{17-18}$ However, routine pretreatment GRT in ART-naïve patients is lacking in Taiwan because of financial constraints.

Informed strategies to prevent the development of virologic failure to first-line ART and the emergence of HIV resistance are, therefore, crucial. Although studies have been conducted on the prevalence of HIV-transmitted drug resistance in northern Taiwan, ${ }^{17,18}$ data on the impact of epidemiologic information and drug resistance after failure to ART are unknown. This study aims to establish the prevalence of genotypic drug resistance after failure of anti-HIV therapy and to establish the clinical and viral characteristics that might be predictive of drug resistance.

\section{Materials and methods Ethical statement}

The institutional review board of the Kaohsiung Veterans General Hospital in Taiwan approved this study (VGHKS98CT1-08 and VGHKS13-CT4-12). The protocol complied with all ethical considerations involving human subjects, and all information obtained followed standard clinical guidelines. All of the study participants provided written informed consent.

\section{Study design and participants}

Samples covering the period 2009-2014 were obtained from a clinical HIV cohort on HIV drug resistance in adult patients at the Veterans General Hospital Surveillance and Monitor Network (VGHSMN), which consisted of branches in Taipei, Taichung, and Kaohsiung, as well as 10 veterans and other satellite hospitals. The inclusion criteria were: 1) HIV-1-infected adult aged $\geq 20$ years; 2) HAART initiation between 1 January 2009 and 31 December 2014; and 3) treatment failure with HIV-1 viral load $\geq 1000$ copies/mL. A standardized questionnaire was also used to collect demographic data that included age, sex, risk factors for HIV infection, CD4 count, viral load, and duration and name of ART used.

Patients with a previous history of ART exposure for prevention of mother to child transmission or for post-exposure prophylaxis were excluded. When the patients returned to the clinics, laboratory exams for CD4 cell counts (FACS Flow; Becton Dickinson and Company, Franklin Lakes, NJ, USA) and plasma viral load (Cobas Amplicor HIV-1 monitor test, version 1.5; Roche Diagnostics Corporation, Indianapolis, IN, USA) were performed. First-line ART was provided for free in designated hospital nationwide by infectious diseases physicians and supported by the HIV case managers. Treatment switches were guided by clinical, immunologic, and virologic criteria, as determined by the physician in charge.

Standard follow-up of HIV-infected patients on ART consisted of outpatient visits every 3 months, with physical examinations, CD4+ T cell count, viral load, hematology, and biochemistry. However, HIV-1 GRT was not routinely performed for patients with virologic failure. Blood samples for such patients could be sent to the Center for Diseases Control for GRT once in every 3 years or to the reference laboratory if the patient was in the VGHSMN cohort.

\section{Genotypic drug resistance testing}

Resistance testing was performed on plasma samples using the ViroSeq ${ }^{\mathrm{TM}}$ HIV-1 Genotyping System version v2.8, according to the manufacturer's instructions (Celera, Alameda, CA, USA). Antiretroviral resistance mutations were defined using the 2015 International Antiviral Society-USA (IAS-USA) HIV drug resistance algorithm, ${ }^{19}$ while drug resistances were compared using the HIVdb program of the Stanford University HIV Drug Resistance Database. Patients were then classified as low-level, intermediate, or high-level resistance, as defined accordingly.

\section{Statistics analysis}

The Mann-Whitney $U$ test was used to compare the median values of continuous variables between groups (resistance and wild virus), while the Fisher's exact test was used to compare categorical variables between the two groups. KaplanMeier curves were estimated to determine the association 
between duration of current ART use and the development of drug resistance. A Cox proportional hazard model was used to calculate the hazard ratio (HR) for drug resistance. A two-sided $p<0.05$ was considered statistically significant. Statistical calculations were performed using the SPSS program version 12.0 (IBM Corporation, Armonk, NY, USA).

\section{Results}

From 2009 to 2014, a total of 359 patients had GRT and sequences were successfully obtained from 290 (81\%). Sixtynine did not report resistance due to low viral load $(n=13)$, poor sample quality $(\mathrm{n}=1)$, and cancellation by the requesting physician due to viral re-suppression after education $(\mathrm{n}=55)$. The demographic data between patients with successful GRT and those without did not show any difference. Among the 359 patients, $93.6 \%(\mathrm{n}=336)$ were male and $69.4 \%(\mathrm{n}=247)$ were aged 20-39 years. Moreover, $73.4 \%(\mathrm{n}=256)$ were men who had sex with men (MSM).

Upon virologic failure, the median CD4 cell count was 214 (interquartile range [IQR]: $71-367)$ cells $/ \mu \mathrm{L}$ and the viral load was $4.5 \log$ (IQR: 3.9-5.0). Most (90\%) of the 290 patients with available GRT report had HIV subtype B, 9\% had CRF01_AE, and 1\% had subtype C (Table 1).

Table I Demographic data among HIV-I infected patients with treatment failure $(\mathrm{N}=359)$

\begin{tabular}{|c|c|}
\hline Parameters & Number of patients (\%) \\
\hline \multicolumn{2}{|l|}{$\operatorname{Sex}(N=359)$} \\
\hline Male & $336(93.6)$ \\
\hline Female & $23(6.4)$ \\
\hline \multicolumn{2}{|l|}{ Age $(N=356)$} \\
\hline $20-29$ & $120(33.7)$ \\
\hline $30-39$ & $127(35.7)$ \\
\hline $40-49$ & $71(19.9)$ \\
\hline$>50$ & $38(10.7)$ \\
\hline \multicolumn{2}{|c|}{ Risk factor $(n=349)$} \\
\hline Heterosexual & $63(18.1)$ \\
\hline MSM & $256(73.4)$ \\
\hline IDU & $30(8.5)$ \\
\hline \multicolumn{2}{|c|}{ Any class of resistance $(n=290)$} \\
\hline Yes & $219(75.5)$ \\
\hline No & $71(24.5)$ \\
\hline \multicolumn{2}{|c|}{ HIV subtype $(n=290)$} \\
\hline Non-B & $29(10)$ \\
\hline B & $261(90)$ \\
\hline \multicolumn{2}{|c|}{ CD4 (cells/ $\mu \mathrm{L})$} \\
\hline Minimum & I \\
\hline Maximum & 1217 \\
\hline Median (IQR) & $214(7 \mid-367)$ \\
\hline
\end{tabular}

(Continued)
Table I (Continued)

\begin{tabular}{|c|c|}
\hline Parameters & Number of patients (\%) \\
\hline \multicolumn{2}{|c|}{ 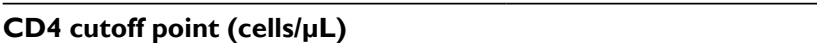 } \\
\hline$\geq 200$ & $189(52.6)$ \\
\hline$<200$ & $170(47.4)$ \\
\hline \multicolumn{2}{|c|}{ Viral load (log) } \\
\hline Minimum & 1.30 \\
\hline Maximum & 6.82 \\
\hline Median (IQR) & $4.53(3.94-4.99)$ \\
\hline \multicolumn{2}{|c|}{ Viral load cutoff point (log) } \\
\hline$\geq 4$ & $258(73.1)$ \\
\hline$<4$ & $95(26.9)$ \\
\hline \multicolumn{2}{|c|}{ Syphilis ( $n=63)$} \\
\hline Negative & $35(55.6)$ \\
\hline Positive & $28(44.4)$ \\
\hline \multicolumn{2}{|c|}{ Months on HAART } \\
\hline Minimum & 0.5 \\
\hline Maximum & 168 \\
\hline Median (IQR) & $24(9-5 I)$ \\
\hline \multicolumn{2}{|c|}{ Months of current regimen } \\
\hline Minimum & 0.1 \\
\hline Maximum & 120 \\
\hline Median (IQR) & $9(4-20.3)$ \\
\hline \multicolumn{2}{|c|}{ Current regimens (CR) } \\
\hline NNRTI based & $158(49.8)$ \\
\hline PI based & $159(50.2)$ \\
\hline \multicolumn{2}{|l|}{ NRTIs in CR } \\
\hline ZDV/3TC & $\mid 35(4 \mid .0)$ \\
\hline $\mathrm{ABC} / 3 \mathrm{TC}$ & $121(36.8)$ \\
\hline TDF/3TC & $31(9.4)$ \\
\hline Others & $42(12.8)$ \\
\hline \multicolumn{2}{|l|}{ NNRTI in CR } \\
\hline NVP & 87 (53.7) \\
\hline EFV & $75(46.3)$ \\
\hline \multicolumn{2}{|l|}{ PIs in CR } \\
\hline Boosted PI & $109(66.9)$ \\
\hline Unboosted PI & $54(33.1)$ \\
\hline \multicolumn{2}{|c|}{ NRTIs in initial regimens (IR) } \\
\hline ZDV/3TC & $152(51.9)$ \\
\hline $\mathrm{ABC} / 3 \mathrm{TC}$ & $98(33.4)$ \\
\hline TDF/3TC & $14(4.8)$ \\
\hline Others & $29(9.9)$ \\
\hline \multicolumn{2}{|l|}{ NNRTIs in IR } \\
\hline NVP & $80(44.7)$ \\
\hline EFV & $99(55.3)$ \\
\hline \multicolumn{2}{|l|}{ PIs in IR } \\
\hline Boosted PI & $85(71.4)$ \\
\hline Unboosted PI & $34(28.6)$ \\
\hline
\end{tabular}

Notes: Pls in CR: Boosted PI consisted of lopinavir/ritonavir ( $n=77)$, atazanavir/ ritonavir $(n=21)$, darunavir/ritonavir $(n=10)$, and tipranavir/ritonavir $(n=1)$. Unboosted PI consisted of atazanavir ( $n=54)$. Pls in IR: Boosted PI consisted of lopinavir/ritonavir $(n=70)$, atazanavir/ritonavir $(n=9)$, indinavir/ritonavir $(n=4)$, and nelfinavir/ritonavir $(n=2)$. Unboosted PI consisted of atazanavir $(n=31)$, indinavir $(n=2)$, and ritonavir $(n=1)$.

Abbreviations: $A B C / 3 T C$, abacavir/lamivudine; EFV, efavirenz; HAART, highly active antiretroviral therapy; HIV, human immunodeficiency virus; IDU, intravenous drug abuser; IQR: interquartile range; MSM, men who have sex with men; NNRTI, non-nucleoside reverse transcriptase inhibitor; NRTI, nucleoside reverse transcriptase inhibitor; NVP, nevirapine; Pl, protease inhibitor; TDF/3TC, tenofovir/ lamivudine; ZDT/3TC, zidovudine/lamivudine; IR, initial regimens; CR, current regimens. 
The median duration of HAART use was 24 months (IQR: 9-50.5 months). The median duration of current HAART upon presentation for GRT was 9 months (IQR: 4-20.3 months). Upon presentation for GRT, 49.8\% (158/317) of the patients were under non-nucleoside reverse transcriptase inhibitor (NNRTI)-based treatment. The most commonly used nucleoside reverse transcriptase inhibitor (NRTI) backbone included zidovudine/lamivudine (41\%), abacavir/ lamivudine (36.8\%), and tenofovir/lamivudine (9.4\%). The most commonly used NNRTIs were nevirapine $(53.7 \%$, $87 / 162)$ and efavirenz $(46.3 \%, 75 / 162)$. Booster protease inhibitor (PI) was used in $109(66.9 \%, 109 / 163)$ patients with lopinavir/ritonavir (47\%, 77/163), atazanavir/ritonavir (13\%, $21 / 163)$, darunavir/ritonavir $(6 \%, 10 / 163)$, and tipranavir/ ritonavir $(0.6 \%, 1 / 163)$. Unboosted PI (atazanavir) was used in $54(33.1 \%)$ patients.

Of the 290 patients with GRT, $75.5 \%(n=219)$ had drug resistance to any of the three classes of antiretroviral drugs, including $58.6 \%$ showing resistance to NRTI, $61.4 \%$ to NNRTI, and $11.4 \%$ to PI (Figure 1). The NNRTI crossresistance was common in $51.7 \%$ of patients who were also resistant to rilpivirine, although they had no previous exposure to this new second-generation NNRTI (Figure 2). The prevalence of IAS-USA drug resistance associated mutations in NRTI, NNRTI, and PI was $62.1 \%, 72.1 \%$, and $37.2 \%$, respectively (Figure 1). The most common NRTI drug resistance associated mutations were M184V (52.1\%), L74V (13.8\%), and Y115F (7.2\%). For NNRTI, the most common drug resistance associated mutations were K103N (26.6\%), Y181C (16.2\%), V179D (14.8\%), and G190A (13.4\%), while for PI, these were A71V (13.1\%), L10I (12.4\%), A71T (11.0\%), and I50L (5.9\%), as shown in Figure 3.
Risk factors associated with the presence of drugresistant strains in a single variance analysis were younger age $(p=0.045)$, MSM (adjusted HR [aHR]: 2.171, 95\% CI: $1.210-3.894 ; p=0.011)$, and NNRTI-based HARRT at failure (aHR: 4.706, 95\% CI: 2.331-9.499; $p<0.0001$ ), as shown in Table 2. Risk factors for any class drug resistance in multivariate analysis included age $\leq 35$ years (aHR: 2.30 , CI: $1.48-3.56 ; p<0.0001)$, initial NNRTI-based antiretroviral (ARV) regimens (aHR: 1.70, CI: $1.10-2.63 ; p=0.018$ ), and currently used NNRTI-based ARV when there is the occurrence of virological failure (odds ratio: 4.04, CI: 2.47-6.59; $p<0.001)$, as shown in Figure 4. There were no associations among HIV-1 subtype, viral load, and resistance (Table 3).

\section{Discussion}

This study illustrates the prevalence of HIV drug resistance among HIV-infected patients with virologic failure after firstline ART in Taiwan. In particular, this study highlights the high rate of drug resistance $(75.5 \%)$ and the association of younger age ( $<35$ years), even with widely available routine viral load monitoring. More importantly, the drug resistance to tenofovir and PI is low, compared to that of NNRTIs.

The findings here are similar to the resistance data from those patients in Africa who have suffered from early failures to the first-line treatment, showing that $70 \%$ with more than one drug resistance mutation after 12 months of treatment. ${ }^{10,20}$ Compared to other studies, the patients here had been a long duration on ART, with a median of 24 months. ${ }^{20}$ Thus, the high prevalence of resistance in this study is likely due to a limited availability of resistance testing, leading to prolonged failure of ART and an accumulation of resistance mutations.

Most of the NRTI backbone used in this study included lamivudine and zidovudine: $52 \%(n=152)$, in the initial regimen

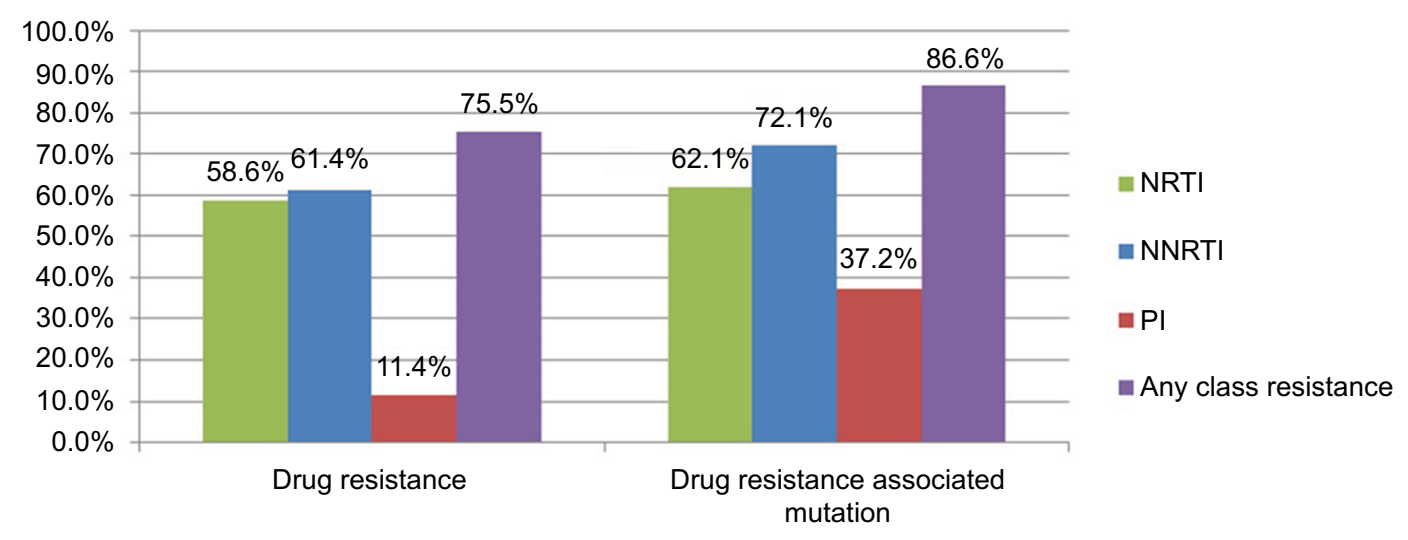

Figure I Percentage of IAS-USA HIV drug resistance associated mutations and drug resistance by HIVdb program of the Stanford University among 290 HIV-I infected patients with virologic failure, 2009-20I4.

Note: A high of $75.5 \%$ of patients had drug resistance to any of the three classes of ART and $86.6 \%$ of the patients harbored any of the drug resistance associated mutations. Abbreviations: ART, antiretroviral therapy; HIV, human immunodeficiency virus; NNRTI, non-nucleoside reverse transcriptase inhibitor; NRTI, nucleoside reverse transcriptase inhibitor; PI, protease inhibitor. 


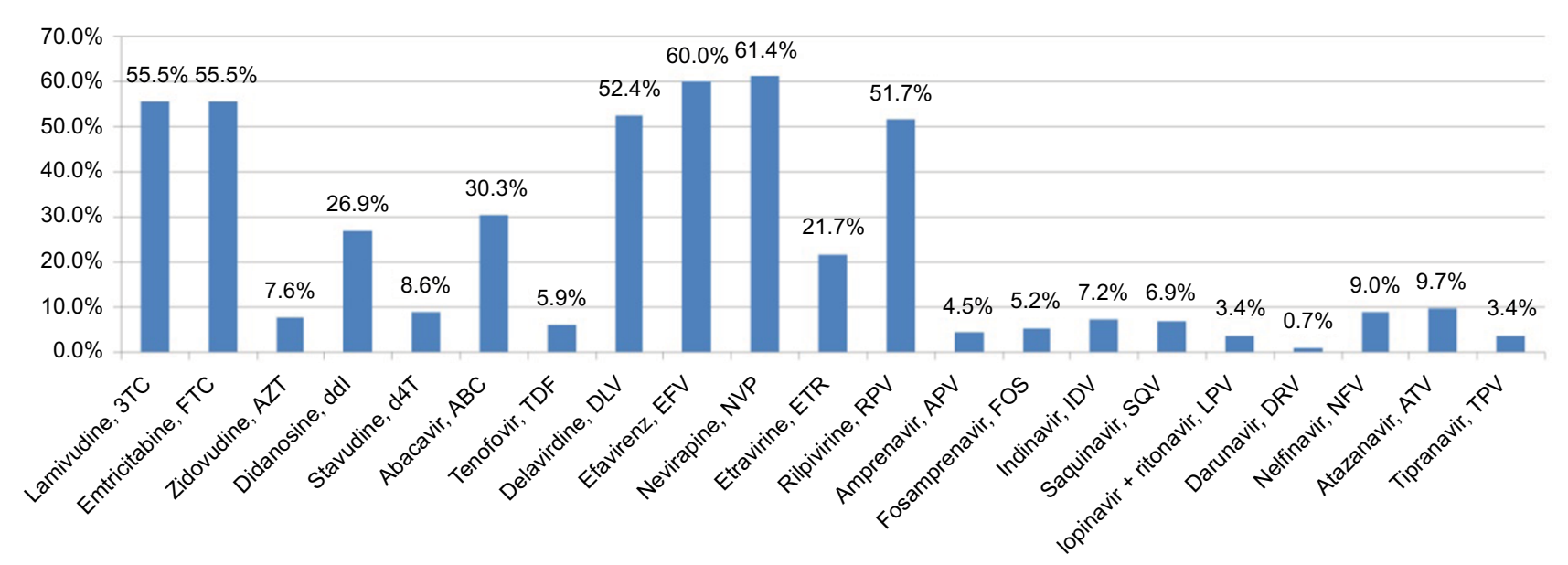

Figure 2 The prevalence of drug resistance to NRTI, NNRTI, and PI among 290 HIV-I infected patients with virologic failure.

Note: Only $5.9 \%$ of the patients had drug resistance to tenofovir. The NNRTI cross-resistance was common and $5 \mathrm{I} .7 \%$ of patients were also resistant to rilpivirine although they had no previous exposure to this new second-generation NNRTI.

Abbreviations: HIV, human immunodeficiency virus; NNRTI, non-nucleoside reverse transcriptase inhibitor; NRTI, nucleoside reverse transcriptase inhibitor; PI, protease inhibitor.

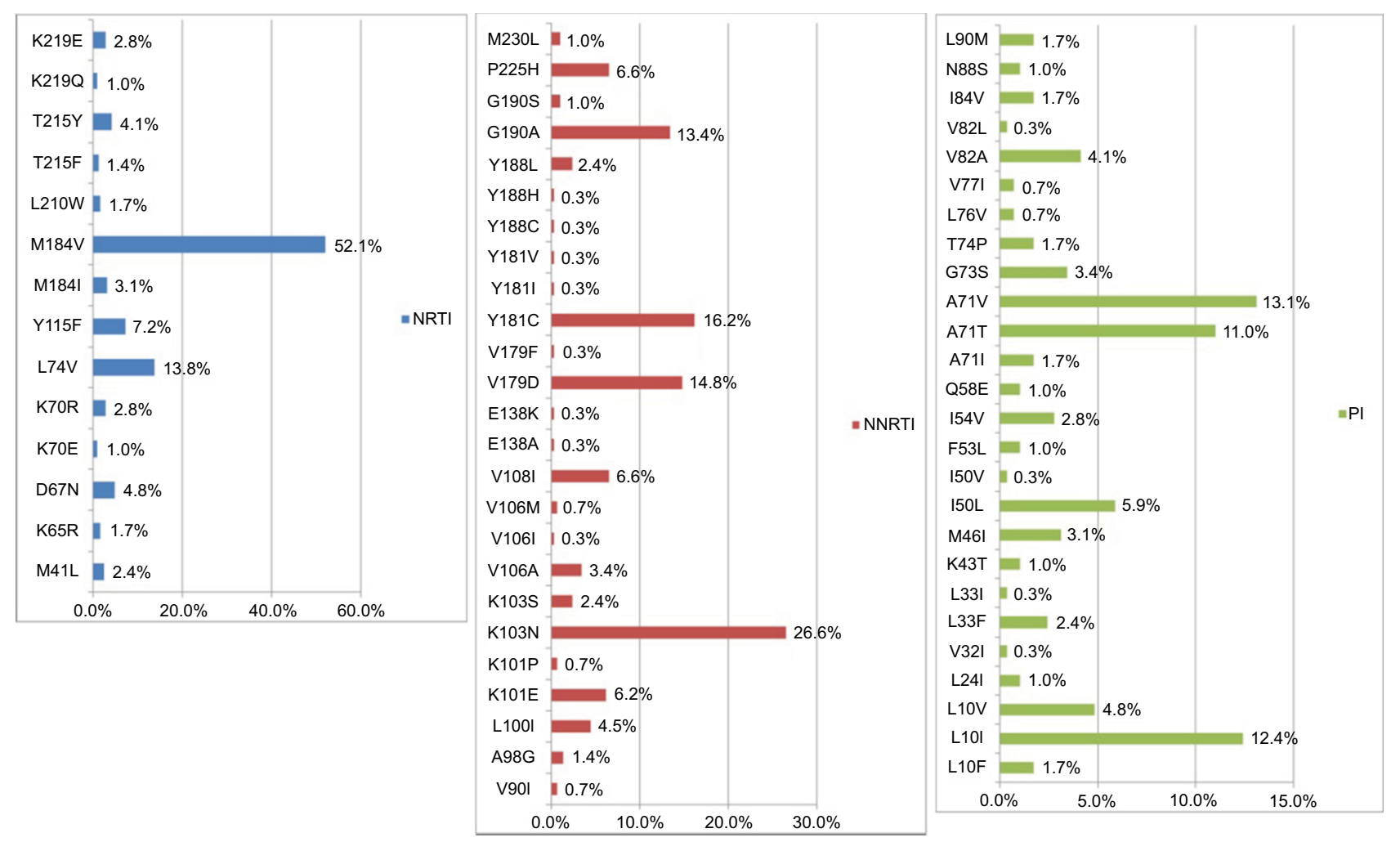

Figure 3 Percentage of ISA-USA HIV drug resistance associated mutations in NRTI, NNRTI, and PI among 290 HIV-I infected patients with virologic failure.

Notes: The most common NRTI drug resistance associated mutations were MI84V (52.1\%) and L74V (I3.8\%). For NNRTI, the most common drug resistance associated mutations were $\mathrm{KIO} \mathrm{N}$ (26.6\%) and YI8IC, while for PI, these were A7IV (I3.I\%) and LIOI (I2.4\%).

Abbreviations: HIV, human immunodeficiency virus; NNRTI, non-nucleoside reverse transcriptase inhibitor; NRTI, nucleoside reverse transcriptase inhibitor; PI, protease inhibitor.

when starting ART and $41 \%(n=135)$ in the current regimen when virologic failure occurred, explaining the predominance of M184V mutations and thymidine-associated mutations at the point of failure. In this study, the K65R mutation (1.7\%) was rare, probably because tenofovir had been introduced in
Taiwan in 2011 and had a restricted use in the national HIV treatment guideline initially. These results suggest that tenofovir remains a good option for second-line therapy.

The prevalence of resistance to NNRTIs was high, which was $60 \%$ for efavirenz and $61.4 \%$ for nevirapine. These 
Table 2 Risk factors associated with HIV-I drug resistance in univariate analysis

\begin{tabular}{|c|c|c|c|c|c|}
\hline Demographic data and drug regimens & $\begin{array}{l}\text { Resistance } \\
(n=219)\end{array}$ & $\begin{array}{l}\text { Nonresistance } \\
(n=7 \text { I) }\end{array}$ & p-value & aHR & $95 \% \mathrm{Cl}$ \\
\hline \multicolumn{6}{|l|}{ Sex } \\
\hline Male & $206(94)$ & $65(92)$ & 0.422 & 1.463 & $0.535-4.003$ \\
\hline Female & $13(6)$ & $6(8)$ & & & \\
\hline Age (median, IQR) & $33(27-4 I)$ & $34(30-44)$ & $0.045^{*}$ & & \\
\hline \multicolumn{6}{|l|}{ Risk factor, n (\%) } \\
\hline MSM & 169 (79) & $44(63)$ & $0.011 *$ & 2.171 & $1.210-3.894$ \\
\hline Non-MSM & $46(21)$ & $26(37)$ & & & \\
\hline Viral load (log) (median, IQR) & $4.6(4.0-5.0)$ & $4.7(4.2-5.3)$ & 0.085 & & \\
\hline CD4 (median, IQR) & $198(58-364)$ & $219(58-343)$ & 0.883 & & \\
\hline \multicolumn{6}{|l|}{ HIV subtype, n (\%) } \\
\hline B & 195(89) & $66(93)$ & 0.495 & 0.612 & $0.225-1.670$ \\
\hline Non-B & $24(\mathrm{II})$ & $5(7)$ & & & \\
\hline \multicolumn{6}{|l|}{ Current regimen, $\mathrm{n}(\%)$} \\
\hline NNRTI based & $120(59)$ & $12(23)$ & $<0.000 I^{*}$ & 4.706 & $2.331-9.499$ \\
\hline PI based & $85(4 I)$ & $40(77)$ & & & \\
\hline \multicolumn{6}{|l|}{ NRTI of current regimen } \\
\hline ZDT/3TC & $80(38)$ & $21(37)$ & & & \\
\hline $\mathrm{ABC} / 3 \mathrm{TC}$ & $80(38)$ & $20(36)$ & & & \\
\hline TDF/3TC & $25(12)$ & $4(7)$ & & & \\
\hline Others & $23(11)$ & II (20) & & & \\
\hline \multicolumn{6}{|l|}{ NNRTI of current regimen } \\
\hline NVP & $69(57)$ & $7(54)$ & 1.000 & 1.16 & $0.354-3.516$ \\
\hline EFV & $53(43)$ & $6(46)$ & & & \\
\hline \multicolumn{6}{|l|}{ Pls in current regimen ${ }^{a}$} \\
\hline Boosted PI & $52(60)$ & $34(83)$ & $0.009 *$ & 0.306 & $0.122-0.767$ \\
\hline Unboosted PI & $35(40)$ & $7(17)$ & & & \\
\hline Months on HAART (median, IQR) & $19(8-52.5)$ & $32(15-48)$ & 0.157 & & \\
\hline Months on current regimen (median, IQR) & $9(5-20)$ & $8.5(3-19.8)$ & 0.599 & & \\
\hline \multicolumn{6}{|l|}{ NRTIs in initial regimen } \\
\hline ZDT/3TC & $86(47)$ & $31(64)$ & & & \\
\hline $\mathrm{ABC} / 3 \mathrm{TC}$ & $71(39)$ & $12(24)$ & & & \\
\hline TDF/3TC & $11(6)$ & I (2) & & & \\
\hline Others & $15(8)$ & $5(10)$ & & & \\
\hline \multicolumn{6}{|l|}{ NNRTIs in initial regimen } \\
\hline NVP & $63(49)$ & $8(36)$ & 0.356 & 1.696 & $0.666-4.321$ \\
\hline EFV & $65(5 I)$ & $14(64)$ & & & \\
\hline \multicolumn{6}{|l|}{ Pls in initial regimen ${ }^{b}$} \\
\hline Boosted PI & $40(68)$ & $22(79)$ & 0.447 & 0.574 & $0.200-1.649$ \\
\hline Unboosted PI & $19(32)$ & $6(21)$ & & & \\
\hline Syphilis & $27 / 59(46)$ & I/4 (25) & 0.622 & 2.531 & $0.249-25.768$ \\
\hline
\end{tabular}

Notes: ${ }^{a}$ For patients who developed resistance, the current use of boosted PI when failure occurred consisted of lopinavir/ritonavir ( $\left.n=29\right)$, atazanavir/ritonavir ( $\mathrm{n}=\mathrm{I5}$ ), darunavir/ritonavir $(n=7)$, and tipranavir/ritonavir $(n=1)$. For patients who developed resistance, the current use of unboosted PI when failure occurred consisted of atazanavir $(n=35)$. For patients who did not develop resistance, the current use of boosted PI when failure occurred consisted of lopinavir/ritonavir $(n=3 \mathrm{I})$, atazanavir/ritonavir $(n=2)$, and darunavir/ritonavir $(n=1)$. For patients who did not develop resistance, the current use of unboosted PI when failure occurred consisted of atazanavir ( $\mathrm{n}=7$ ). ${ }^{\mathrm{b}}$ For patients who developed resistance, the initial regimen of boosted PI when failure occurred consisted of lopinavir/ritonavir $(n=32)$, atazanavir/ritonavir ( $n=6)$, and indinavir/ritonavir $(n=2)$. For patients who developed resistance, the initial regimen of unboosted PI when failure occurred consisted of atazanavir $(n=I 6)$, indinavir $(n=2)$, and ritonavir $(n=I)$. For patients who did not develop resistance, the initial regimen of boosted PI when failure occurred consisted of lopinavir/ritonavir $(n=20)$ and atazanavir/ritonavir ( $n=2$ ). For patients who did not develop resistance, the initial regimen of unboosted PI when failure occurred consisted of atazanavir $(n=6)$. ${ }^{*}<0.05$.

Abbreviations: aHR, adjusted hazard ratio; ABC/3TC, abacavir/lamivudine; EFV, efavirenz; HAART, highly active antiretroviral therapy; HIV, human immunodeficiency virus; IQR, interquartile range; MSM, men who have sex with men; NNRTI, non-nucleoside reverse transcriptase inhibitor; NRTI, nucleoside reverse transcriptase inhibitor; NVP, nevirapine; PI, protease inhibitor; TDF/3TC, tenofovir/lamivudine; ZDT/3TC, zidovudine/lamivudine.

were similar to the results of studies reported from Africa, where a high rate of resistance to first-generation NNRTIs was observed. ${ }^{10,20}$ In the present cohort, initial and current NNRTI-based ARV was associated with the development of drug resistance and only $78.3 \%$ and $48.3 \%$ of the individual genotypes predicted full susceptibility to etravirine and rilpivirine, respectively. None of the patients here had a previous exposure to rilpivirine or etravirine. These results also impacted on the second-generation NNRTI drug choices in patients with treatment failure. Studies show that nevirapine 


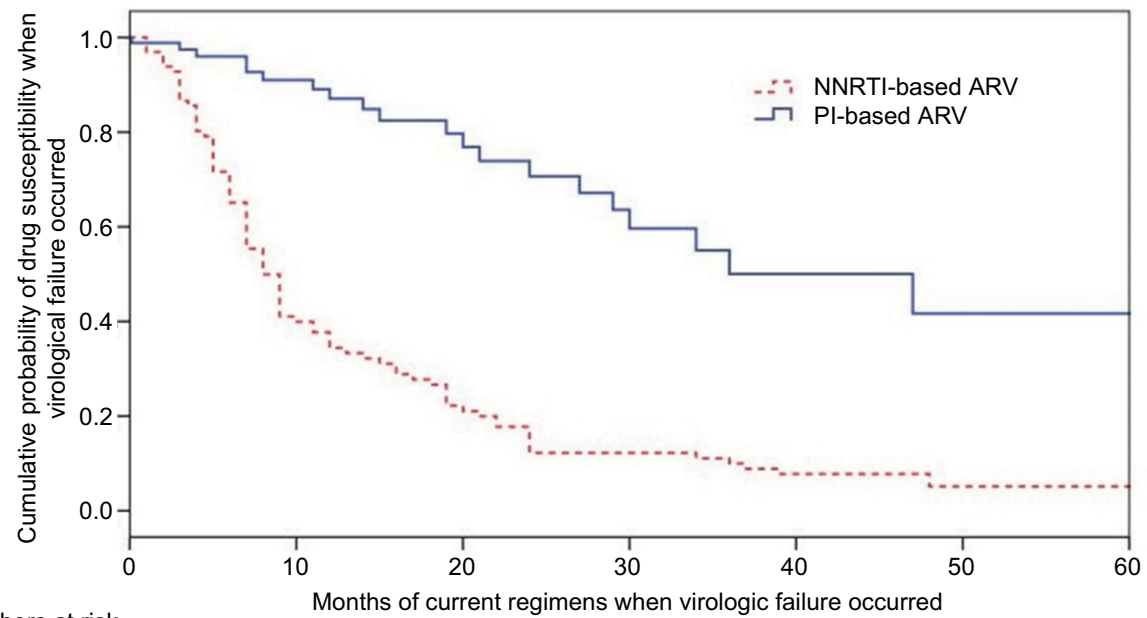

Numbers at risk

Months of current regimens when virologic failure occurred

$\begin{array}{rrrrrrrr}\text { NNRTI based } & 74 & 23 & 9 & 6 & 4 & 0 & 5 \\ \text { PI-based } & 50 & 24 & 13 & 15 & 4 & 5 & 2\end{array}$

Figure 4 Kaplan-Meier curves for probabilities of developing drug resistances in patients after failure of their current regimen ( $p<0.000 \mathrm{I}$, log rank test).

Notes: Patients with NNRTI-based regimen were more likely to develop virologic failure, compared to those on PI-based regimens (odds ratio: 4.04 , Cl: $2.47-6.59$; $p<0.001$ ). The numbers used in the category at risk were 234 . However, 290 samples were successfully tested for resistance. The detailed information for the drug prescription was not available in 56 subjects.

Abbreviations: NNRTI, non-nucleoside reverse transcriptase inhibitor; NRTI, nucleoside reverse transcriptase inhibitor; PI, protease inhibitor.

Table 3 Risk factors associated with drug resistance in Cox regression model

\begin{tabular}{|c|c|c|c|c|c|}
\hline \multirow[t]{2}{*}{ Variable } & \multirow[t]{2}{*}{ Number (\%) } & \multicolumn{2}{|c|}{ Univariate analysis } & \multicolumn{2}{|c|}{ Multivariate analysis } \\
\hline & & HR (95\% CI) & $p$-value & $\operatorname{aHR}(95 \% \mathrm{Cl})$ & $p$-value \\
\hline Total & 290 & & & & \\
\hline \multicolumn{6}{|l|}{ Sex } \\
\hline Male & 271 (93.4) & I.2I (0.53-2.76) & 0.65 & & \\
\hline Female & $19(6.6)$ & 1 & & & \\
\hline \multicolumn{6}{|l|}{ Age (years) } \\
\hline$\leq 35$ & $176(60.7)$ & $2.49(1.64-3.79)$ & $<0.0001$ & $2.30(1.48-3.56)$ & $<0.0001$ \\
\hline$>36$ & $112(38.6)$ & 1 & & & \\
\hline \multicolumn{6}{|c|}{ Route of transmission } \\
\hline MSM & $213(73.4)$ & $1.62(0.99-2.65)$ & 0.054 & & \\
\hline Non-MSM & $72(24.8)$ & 1 & & & \\
\hline \multicolumn{6}{|c|}{ HIV RNA (copies/mL) } \\
\hline$\leq 10,000$ & $219(75.5)$ & I.44 (0.86-2.40) & 0.17 & & \\
\hline$>10,000$ & $65(22.4)$ & 1 & & & \\
\hline \multicolumn{6}{|c|}{ CD4 count (cells/ $\mu \mathrm{L})$} \\
\hline$\leq 200$ & $235(8 I)$ & I.09 (0.70-I.7I) & 0.70 & & \\
\hline$>200$ & $55(19)$ & 1 & & & \\
\hline \multicolumn{6}{|c|}{ Initial ART regimen } \\
\hline NNRTI & $150(5 \mid .7)$ & $1.90(1.25-2.90)$ & 0.003 & $1.70(1.10-2.63)$ & 0.018 \\
\hline PI & $87(30)$ & $0.46(0.30-0.72)$ & $<0.0001$ & $0.51(0.32-0.80)$ & 0.003 \\
\hline \multicolumn{6}{|c|}{ Current ART regimen } \\
\hline NNRTI & $135(46.6)$ & $4.5 ।(2.8-7.28)$ & $<0.0001$ & $4.04(2.47-6.59)$ & $<0.0001$ \\
\hline $\mathrm{PI}$ & $128(44.1)$ & $0.23(0.14-0.37)$ & $<0.0001$ & $0.26(0.16-0.42)$ & $<0.0001$ \\
\hline \multicolumn{6}{|l|}{ HIV strain } \\
\hline Subtype B & $260(89.7)$ & I.4I (0.78-2.53) & 0.26 & & \\
\hline Non-subtype B & $29(10)$ & 1 & & & \\
\hline
\end{tabular}

Notes: Statistical analysis: Variables with a $p$ value $<0.20$ in the univariate analysis were considered for inclusion in multivariate Cox regression models. $p$ Values $<0.05$ were considered statistically significant. All analyses were performed using SPSS software version 12 .

Abbreviations: aHR, adjusted hazard ratio; ART, antiretroviral therapy; HIV, human immunodeficiency virus; HR, hazard ratio; MSM, men who have sex with men; NNRTI, non-nucleoside reverse transcriptase inhibitor; PI, protease inhibitor. 
selects for the Y181C and G190A mutations and leads to reduced rilpivirine or etravirine susceptibility. ${ }^{21,22}$ Efavirenz failure is more likely to be associated with K103N mutation and has little impact on etravirine susceptibility. ${ }^{21,22}$ The median duration for the development of NNRTI resistance was 9 months, reflecting the low genetic barrier nature of NNRTI and poor drug compliance in the younger age group.

In this study, only $11.4 \%$ of patients developed PI resistance. The emergence of PI resistance at the time of virologic failure is uncommon in PI-naïve patients who experience virologic failure during their PI regimen. Moreover, the PI mutations can be located outside the pol gene, thereby underestimating the prevalence of PI resistance. ${ }^{23-25}$ In previous studies in Taiwan, the transmitted drug resistance to PI was extremely low $(<4 \%) \cdot{ }^{17,18}$ The PIs used in this study with failure were mostly lopinavir/ritonavir (60/125, 48\%) and unboosted atazanavir $(42 / 125,33.6 \%)$. Up to $88.6 \%$ of patients did not have PI resistance on treatment failure and there was no association between unboosted PI use and the development of resistance. This might be due to the poor access to GRT and poor drug adherence.

Rates of PI resistance at the time of second-line failure are high in resource-limited settings and are associated with the duration of exposure to previous drug regimens and poor adherence. ${ }^{26} \mathrm{~A}$ high prevalence of PI resistance was reported in four studies, two of which studied patients from Asia, especially from India $(n=45,73 \%)^{27}$ and Vietnam $(n=231$, $59 \%) .{ }^{28}$ The other two studies were from Mali $(n=93,25 \%)^{29}$ and Nigeria $(n=61,62 \%) \cdot{ }^{30}$ Except for the study from India, where indinavir/ritonavir and atazanavir/ritonavir were used, the studies in these other countries used lopinavir/ritonavir for second-line therapy, similar to this study.

In this study, younger age ( $<35$ years) is associated with the development of resistance. Several large cohort studies also report that older age is associated with better treatment outcomes, ${ }^{31,32}$ although better adherence does not necessarily explain the lower risk of failure in this group. No data about the level of adherence was available in this study. However, $24.5 \%$ of patients who experienced virologic failure had the wild-type virus, suggesting that adherence was an important factor in virologic failure.

The resistance rates to any of the three classes of antiretroviral drugs (NRTI, NNRTI, and PI) were up to $75.5 \%$, despite the viral load measurements taken every 3-4 months. This rate was similar to those of developing countries in which viral load monitoring was not routinely available. In an HIV clinical trial in the USA where viral load was measured regularly every 3 months, the frequency of resistance in the study subjects receiving optimal first-line regimens was $62 \%$ at virologic failure..$^{33}$ This was similar to the $70 \%$ drug resistance rate in the Pharm Access African Studies to Evaluate Resistance, where HIV plasma viral load was measured after 12 months of a first-line regimen. ${ }^{10}$ In the ANRS (National Agency for AIDS Research) 1268 study, the overall prevalence of major drug resistance mutations after virologic failure without any RNA monitoring was $71 \%$ and $86 \%$ among those assessed after 1 and 2 years, respectively, consistent with the evidence of a modest increase in resistance without monitoring among recipients of first-line treatment in Africa. ${ }^{34-36}$

It is very difficult to ascribe rates of virologic failure in resource-limited settings to a lack of viral load monitoring. Patient treatment adherence, HAART regimens used, accessibility of genotypic drug resistance, duration of failing regimen before resistance testing, even challenges in providing sample transport, laboratory and clinical infrastructure, and trained personnel, ${ }^{10}$ and competing for treatment resources all play significant roles that weaken the efficiency of routine viral load monitoring in the prevalence of drug resistance.

\section{Limitations}

There was no data available on the baseline prevalence of HIVtransmitted drug resistance for the study population. Nonetheless, the prevalence of transmitted antiretroviral resistance was reported to be around $8 \%-10 \%$ in Taiwan. The duration of virologic failure under treatment was not precisely determined in all of the patients because the information was lacking in some of the patients. In patients failing ART, GRT was found to have a significant benefit on the virologic response when choosing the salvage regimens. ${ }^{37-39}$ But the treatment outcomes for patients with virologic failures switching to another regimen were not available, making it impossible to evaluate the clinical impact of GRT. There was also no information on adherence to the ART regimen, making interpretation of the effect of age on the development of resistance more complicated. However, several large cohort studies reported that older age was associated with better treatment outcomes, although better adherence did not necessarily explain the lower risk of treatment failure in this group. Lastly, the results were from the VGHSMN cohort. Thus, it would be interesting to extend this work to the other parts of Taiwan.

\section{Conclusion}

This study demonstrates a high level of resistance to NRTI and NNRTI among patients who experienced virologic failure to first-line antiretroviral therapy even with routine viral load monitoring. It is crucial to educate younger MSM individuals to maintain good adherence to their treatment regimen. The use of PI is also associated with lower possibility of drug resistance. 


\section{Acknowledgments}

The authors thank Dr Wing-Wai Wong, Department of Infectious Diseases, Taipei Veterans General Hospital and Dr Yu-Huei, Lin, Department of Infectious Diseases, Taichung Veterans General Hospital, Taiwan for their help. This study was funded by the Medical Foundation in Memory of Dr DehLin Cheng (MF-DLC100053S5C2Y1) and by the Veterans General Hospitals and University System of Taiwan Joint Research Program Grant (VGHUST105-G3-2-1).

\section{Disclosure}

The authors report no conflicts of interest in this work.

\section{References}

1. Sterne JA, Hernan MA, Ledergerber B, et al; Egger M;Swiss HIV Cohort Study. Long-term effectiveness of potent anti-retroviral therapy in preventing AIDS and death: a prospective cohort study. Lancet. 2005;366(9483):378-384.

2. Bor J, Herbst AJ, Newell ML, Bärnighausen T. Increases in adult life expectancy in rural South Africa: valuing the scale-up of HIV treatment Science. 2013;339(6122):961-965.

3. Jin Y, Liu Z, Wang X, et al. A systematic review of cohort studies of the quality of life in HIV/AIDS patients after anti-retroviral therapy. Int J STD AIDS. 2014;25(11):771-777.

4. Kumarasamy N, Solomon S, Chaguturu SK, et al. The changing natural history of HIV disease: before and after the introduction of generic anti-retroviral therapy in southern India. Clin Infect Dis. 2005;41(10):1525-1528.

5. Katzenstein DA. HIV RNA and genotype in resource-limited settings: can we do better? Clin Infect Dis. 2014;58(1):110-112.

6. Rupérez M, Pou C, Maculuve S, et al. Determinants of virological failure and anti-retroviral drug resistance in Mozambique. J Antimicrob Chemother. 2015;70(9):2639-2647.

7. World Health Organization. WHO Global Strategy for the Surveillance and Monitoring of HIV Drug Resistance. 2012. Available from: http:/ apps.who.int/iris/bitstream/10665/77349/1/9789241504768_eng.pdf. Accessed September 11, 2015.

8. Hosseinipour MC, Gupta RK, Van Zyl G, Eron JJ, Nachega JB. Emergence of HIV drug resistance during first- and second-line anti-retroviral therapy in resource limited settings. J Infect Dis. 2013;207 (Suppl 2): S49-S56.

9. Scherrer AU, von Wyl V, Yang WL, et al; and the Swiss HIV Cohort Study. Emergence of acquired HIV-1 drug resistance has almost been stopped in Switzerland - a 15 year prospective cohort analysis. Clin Infect Dis. 2016;62(10):1310-1317

10. Hamers RL, Sigaloff KC, Wensing AM, et al; PharmAccess African Studies to Evaluate Resistance (PASER). Patterns of HIV-1 drug resistance after first-line anti-retroviral therapy (ART) failure in 6 subSaharan African countries: implications for second-line ART strategies. Clin Infect Dis. 2012;54(11):1660-1669.

11. Hunt GM, Ledwaba J, Basson AE, et al. Surveillance of transmitted HIV-1 drug resistance in Gauteng and KwaZulu-Natal Provinces, South Africa, 2005-2009. Clin Infect Dis. 2012;54 (Suppl 4):S334-S338.

12. Wood E, Montaner JS. Time to get serious about HIV anti-retroviral resistance. Lancet Infect Dis. 2011;11(10):723-724.

13. Liao L, Xing H, Shang H, et al. The prevalence of transmitted antiretroviral drug resistance in treatment-naive HIV-infected individuals in China. J Acquir Immune Defic Syndr. 2010;53 (Suppl 1):S10-S14.

14. Hattori J, Shiino T, Gatanaga H, et al. Trends in transmitted drugresistant HIV-1 and demographic characteristics of newly diagnosed patients: nationwide surveillance from 2003 to 2008 in Japan. Antiviral Res. 2010;88(1):72-79.
15. Kim MH, Song JE, Ahn JY, et al. HIV anti-retroviral resistance mutations among anti-retroviral treatment-naive and -experienced patients in South Korea. AIDS Res Hum Retroviruses. 2013;29:1617-1620.

16. Sungkanuparph S, Sukasem C, Kiertiburanakul S, Pasomsub E, Chantratita W. Emergence of HIV-1 drug resistance mutations among anti-retroviral-naïve HIV-1-infected patients after rapid scaling up of anti-retroviral therapy in Thailand. J Int AIDS Soc. 2012;15(1):12.

17. Lai CC, Hung CC, Chen MY, et al. Trends of transmitted drug resistance of HIV-1 and its impact on treatment response to first-line anti-retroviral therapy in Taiwan. J Antimicrob Chemother. 2012;67(5):1254-1260.

18. Lai CC, Liu WC, Fang CT, et al. Transmitted drug resistance of HIV-1 strains among individuals attending voluntary counselling and testing in Taiwan. J Antimicrob Chemother. 2016;71(1):226-234.

19. Wensing AM, Calvez V, Günthard HF et.al. 2015 Update of the Drug resistance Mutations in HIV-1. Top Antivir Med. 2015;23(4): 132-141.

20. Ugbena R, Aberle-Grasse J, Diallo K, et al. Virological response and HIV drug resistance 12 months after antiretroviral therapy initiation at 2 clinics in Nigeria. Clin Infect Dis. 2012;54 (Suppl 4):S375-S380.

21. Tambuyzer L, Nijs S, Daems B, Picchio G, Vingerhoets J. Effect of mutations at position E138 in HIV-1 reverse transcriptase on phenotypic susceptibility and virologic response to etravirine. J Acquir Immune Defic Syndr. 2011;58(1):18-22.

22. Vingerhoets J, Nijs S, Tambuyzer L, Hoogstoel A, Anderson D, Picchio G. Similar predictions of etravirine sensitivity regardless of genotypic testing method used: comparison of available scoring systems. Antivir Ther. 2012;17(8):1571-1579.

23. Nijhuis M, van Maarseveen NM, Lastere S, et al. A novel substratebased HIV-1 protease inhibitor drug resistance mechanism. PLoS Med. 2007;4(1):e36.

24. Larrouy L, Chazallon C, Landman R, et al; and the ANRS 127 Study Group. Gag mutations can impact virological response to dual-boosted protease inhibitor combinations in anti-retroviral-naïve HIV-infected patients. Antimicrob Agents Chemother. 2010;54(7):2910-2919.

25. Fun A, Wensing AM, Verheyen J, Nijhuis M. Human immunodeficiency virus gag and protease: partners in resistance. Retrovirology. 2012;9:63.

26. Ajose O, Mookerjee S, Mills EJ, Boulle A, Ford N. Treatment outcomes of patients on second-line antiretroviral therapy in resourcelimited settings: a systematic review and meta-analysis. AIDS. 2012;26(8):929-938.

27. Saravanan S, Vidya M, Balakrishnan P, et al. Viremia and HIV-1 drug resistance mutations among patients receiving second-line highly active antiretroviral therapy in Chennai, Southern India. Clin Infect Dis. 2012;54(7):995-1000.

28. Thao VP, Quang VM, Day JN, et al. High prevalence of PI resistance in patients failing second-line ART in Vietnam. J Antimicrob Chemother. 2016;71(3):762-774.

29. Maiga AI, Fofana DB, Cisse M, et al. Characterization of HIV-1 antiretroviral drug resistance after second-line treatment failure in Mali, a limited-resources setting. J Antimicrob Chemother. 2012;67(12): 2943-2948.

30. Rawizza HE, Chaplin B, Meloni ST, et al. Accumulation of protease mutations among patients failing second-line anti-retroviral therapy and response to salvage therapy in Nigeria. PLoS One. 2013;8:e73582.

31. Weintrob AC, Fieberg AM, Agan BK, et al. Increasing age at HIV seroconversion from 18 to 40 years is associated with favorable virologic and immunologic responses to HAART. JAcquir Immune Defic Syndr. 2008;49(1):40-47.

32. Grabar S, Kousignian I, Sobel A, et al. Immunologic and clinical responses to highly active anti-retroviral therapy over 50 years of age. Results from the French Hospital Database on HIV. AIDS. 2004;18(15): 2029-2038.

33. Mollan K, Daar ES, Sax PE, et al; and the AIDS Clinical Trials Group Study A5202 Team. HIV-1 amino acid changes among participants with virologic failure: associations with first-line efavirenz or atazanavir plus ritonavir and disease status. J Infect Dis. 2012;206(12):1920-1930. 
34. Bartlett JA, Ribaudo HJ, Wallis CL, et al. Lopinavir/ritonavir monotherapy after virologic failure of first-line anti-retroviral therapy in resource-limited settings. AIDS. 2012;26(11):1345-1354.

35. Orrell C, Walensky RP, Losina E, et al. HIV type-1 clade C resistance genotypes in treatment-naive patients and after first virological failure in a large community anti-retroviral therapy programme. Antivir Ther. 2009; 14:523-531.

36. Sigaloff KC, Ramatsebe T, Viana R, de Wit TF, Wallis CL, Stevens WS. Accumulation of HIV drug resistance mutations in patients failing first-line anti-retroviral treatment in South Africa. AIDS Res Hum Retroviruses. 2012;28(2):171-175.
37. Durant J, Clevenbergh P, Halfon P, et.al. Drug-resistance genotyping in HIV-1 therapy: the VIRADAPT randomised controlled trial. Lancet. 1999;353(9171):2195-2199.

38. Baxter JD, Mayers DL, Wentworth DN, et, al. A randomized study of antiretroviral management based on plasma genotypic antiretroviral resistance testing in patients failing therapy. CPCRA 046 Study Team for the Terry Beirn Community Programs for Clinical Research on AIDS. AIDS. 2000;14(9):F83-F93.

39. Falloon J. Time to genotype for selection of antiretroviral regimens in previously treated patients? Lancet. 1999;353(9171):2173-2174.
Infection and Drug Resistance

\section{Publish your work in this journal}

Infection and Drug Resistance is an international, peer-reviewed openaccess journal that focuses on the optimal treatment of infection (bacterial, fungal and viral) and the development and institution of preventive strategies to minimize the development and spread of resistance. The journal is specifically concerned with the epidemiology of antibiotic
Dovepress

resistance and the mechanisms of resistance development and diffusion in both hospitals and the community. The manuscript management system is completely online and includes a very quick and fair peerreview system, which is all easy to use. Visit http://www.dovepress.com/ testimonials.php to read real quotes from published authors.

Submit your manuscript here: https://www.dovepress.com/infection-and-drug-resistance-journal 